\title{
CONSECUENCIAS PEDAGÓGICAS ENTRE EL ALUMNADO DE ENSEÑANZA BÁSICA DERIVADAS DE LA COVID-19. UNA REFLEXIÓN EN TORNO A LOS GRANDES OLVIDADOS DE LA PANDEMIA
}

\author{
Pedagogical Consequences of COVID-19 among Basic \\ Education Students. A Reflection on the Great Forgotten \\ ones of the Pandemic
}

Roberto SANZ PONCE y Elena LÓPEZ LUJÁN

*Universidad Católica de Valencia. España.

roberto.sanz@ucv.es; elena.lopez@ucv.es

http://orcid.org/0000-0003-1147-743X; https://orcid.org/0000-0002-2196-658X

Fecha de recepción:16/01/2021

Fecha de aceptación: 05/03/2021

Fecha de publicación en línea: 01/07/2021

Cómo citar este artículo: Sanz Ponce, R. y López Luján, E. (2021). Consecuencias pedagógicas entre el alumnado de enseñanza básica derivadas de la Covid-19. Una reflexión en torno a los grandes olvidados de la pandemia. Teoría de la Educación. Revista Interuniversitaria, 33(2), 149-166. https://doi.org/10.14201/teri.25471

RESUMEN

La pandemia de la COVID-19, que trajo consigo un periodo largo de confinamiento y el cierre de escuelas, ha supuesto un duro golpe a multitud de alumnos que se encontraban en situación de vulnerabilidad. Estos estudiantes han tenido dificultades para seguir el ritmo escolar en sus casas por diferentes motivos, de índole pedagógico, económico y/o cultural. Por ello, el objetivo de este artículo es descifrar y analizar el impacto y la realidad de las tres brechas educativas generadas a partir de la COVID-19: la brecha de aprendizaje, la brecha digital y la brecha escolar, para 
posteriormente elaborar una serie de propuestas educativas con el fin de reestablecer la equidad en el sistema educativo. La pérdida de aprendizajes, la no adquisición de ciertas competencias básicas, el desapego por la escuela y por la educación, el aumento de la tasa de abandono escolar, los problemas de índole emocional y psicológico,..., son algunas de las consecuencias que han sufrido y sufren los estudiantes. Estas consecuencias han afectado fundamentalmente a los alumnos más desfavorecidos, aumentando la inequidad del sistema educativo. Por ello, la puesta en marcha de políticas educativas destinadas a paliar estas consecuencias y la implementación de estrategias pedagógicas para reenganchar al alumnado hacia el aprendizaje, como por ejemplo: modificar el curriculum —flexibilizarlo—; desarrollar metodologías más motivadoras; establecer una enseñanza personalizada; potenciar la comunicación y las corresponsabilidad entre familia-escuela; implementar programas de educación emocional; vigilar el abandono educativo y atajar sus causas,..., son esenciales. El papel de los docentes también es muy importante, así como sus cualidades personales y su talante pedagógico. Aprender de los errores cometidos es una necesidad, ya que no es descartable nuevos periodos de confinamiento en los próximos años.

Palabras clave: COVID-19; brecha de aprendizaje; brecha digital; brecha escolar; alumnos desfavorecidos; equidad educativa; cierre de escuelas; confinamiento.

\section{ABSTRACT}

The COVID-19 pandemic, which brought with it a long period of confinement and school closures, has dealt a severe blow to many vulnerable students. These students have had difficulty keeping up with school at home for various reasons, including pedagogical, economic and/or cultural ones. For this reason, the objective of this article is to decipher and analyze the impact and reality of the three educational gaps generated by the COVID-19: the learning gap, the digital gap and the school gap, in order to later develop a series of educational proposals to re-establish equity in the educational system. The loss of learning, the failure to acquire certain basic skills, the detachment from school and education, the increase in the dropout rate, emotional and psychological problems,... are some of the consequences that students have suffered and are suffering. These consequences have fundamentally affected the most disadvantaged students, increasing the inequity of the educational system. Therefore, the implementation of educational policies aimed at mitigating these consequences and the implementation of pedagogical strategies to reengage students in learning, such as: modifying the curriculum - making it more flexible - ; developing more motivating methodologies; establishing personalized teaching; promoting communication and co-responsibility between family and school; implementing emotional education programs; monitoring educational abandonment and tackling its causes,..., are essential. The role of the teachers is also very important, as well as their personal qualities and their pedagogical approach. Learning from the mistakes made is a necessity, since new periods of confinement cannot be ruled out in the coming years.

Key words: COVID-19; learning gap; digital gap; school gap; disadvantaged students; educational equity; school closure; confinement. 


\section{INTRODUCCIÓN}

En septiembre de 2015, 193 países se comprometían en el cumplimiento de los 17 Objetivos de Desarrollo Sostenible que proponía la Organización de Naciones Unidas (ONU) para el año 2030. Se trataba de la conocida Agenda 2030. Este manifiesto, tal y como se recoge en su Preámbulo, intenta ser un nuevo contrato social global, que permita no dejar a ningún ciudadano atrás, abandonado o excluido. En ese sentido, el Objetivo número 1, dedicado al Fin de la pobreza, nos propone:

Garantizar la protección social de las personas en situación de pobreza o vulnerabilidad social, aumentar el acceso a los servicios básicos y ayudar a las personas afectadas por fenómenos climáticos extremos, así como hacer frente a los problemas económicos, sociales y ambientales.

En esa misma línea, el Objetivo número 4, destinado a la consecución de una Educación de calidad, aboga por "garantizar una educación inclusiva y equitativa de calidad y promover oportunidades de aprendizaje permanente para todos.» Parece obvio que la pandemia, provocada por la COVID-19, ha complicado -imposibilitado- que estos Objetivos puedan considerarse realistas hoy en día.

A pesar de que todos los estudios actuales confirman el menor impacto, tanto en la facilidad de contagio como en la gravedad de los síntomas y de las secuelas producidos por la COVID-19 entre la población infantil y juvenil a nivel mundial, sí que parece evidente que son muchas las consecuencias socioeducativas y psicológicas que el virus -entendido como el cierre de las escuelas y el confinamiento posterior- está acarreando y puede acarrear en el futuro en muchos niños y jóvenes, fundamentalmente, en aquellos que pertenecen a grupos sociales vulnerables o a familias con menos recursos. La COVID-19 ha modificado el perfil de la población vulnerable, entendida como el grupo de población en situación de desprotección, riesgo o desventaja. Según el Informe Distancia social y derecho al cuidado (Foessa, 2020), además del perfil tradicional de vulnerabilidad — familias con menores a cargo, numerosas, de carácter monoparental, sustentados por mujeres o por una persona inmigrante-, la pandemia ha puesto de manifiesto una nueva realidad. Tras solo 2 meses (marzo-mayo de 2020) desde la implantación del Decreto del estado de alarma, se observa que: ocho de cada diez familias en situación de exclusión viven en barrios en buenas condiciones, están encabezados por un hombre, de origen español y que tiene trabajo. Esta realidad se ha visto agravada con el paso del tiempo y con las medidas económicas restrictivas que se han ido implantando.

Estas circunstancias han provocado que muchos de estos niños y jóvenes hayan permanecido olvidados, excluidos e invisibles en sus propias casas a la influencia educativa (De Alba, 2020) durante varios meses. Este tiempo olvidados, excluidos e invisibles ha disminuido la "oportunidad de aprender» (Reimers \& Schleicher, 2020, p. 6), es decir, el tiempo dedicado al aprendizaje, lo cual ha disminuido sustancialmente la adquisición de conocimientos y de habilidades necesarias para un 
correcto desempeño vital y puede desencadenar en el aumento de la transmisión intergeneracional de la pobreza (Foessa, 2020). Tal es así que la citada pandemia y el cierre de los centros educativos en España, tras la declaración del estado de alarma (Real Decreto 463/2020, de 14 de marzo), han demostrado, desgraciadamente, que la educación puede servir por igual para fomentar la equidad y la igualdad de oportunidades entre los estudiantes o para todo lo contrario, es decir, para reproducir y potenciar las desigualdades y las brechas de aprendizaje.

Por todo ello, esta crisis, según la estimación realizada por la UNESCO (2020c, p. 1) para los próximos años, «tendrá consecuencias perdurables en los sistemas educativos en términos de acceso, calidad, equidad y gestión, las cuales presumiblemente persistirán una vez concluida la epidemia.» En ese sentido, el Banco Mundial (2020c) nos avisa de la aparición de nuevos perfiles de pobreza a causa de la COVID-19. Los «nuevos pobres» tendrán más estudios, vivirán en zonas urbanas y en países del primer mundo.

Con el fin de paliar estas consecuencias perdurables en los sistemas educativos, nuevamente la UNESCO (2020a, p. 2), en otro de sus Informes, establece una serie de medidas y/o recomendaciones para "propiciar sistemas educativos más inclusivos, equitativos y resilientes," que puedan dar respuesta a los grandes desafíos planteados en el mundo educativo post-COVID-19. Atender a los grupos vulnerables; recuperar los aprendizajes perdidos; potenciar una educación emocional que palie las secuelas del confinamiento; reenganchar a los alumnos en peligro de deserción escolar o de abandono temprano de los estudios; compensar las posibles desigualdades educativas generadas por las diferencias socioculturales y económicas de las familias; ..., son algunos de los grandes retos a los que se enfrentan los sistemas educativos a nivel internacional en la actualidad. Todo ello, sin descuidar las medidas sanitarias oportunas de distanciamiento social, higiene de manos y uso de mascarillas.

En ese sentido, Save the Children (2020, p. 1) se marca como objetivo prioritario «que los niños y niñas en situación de vulnerabilidad puedan ejercer de forma completa su derecho a la educación» teniendo en cuenta las consecuencias derivadas de la pandemia. Se cree y los resultados así lo vienen atestiguando que el impacto de la COVID-19 tiene tres efectos fundamentales. Por un lado, los derivados del cierre de las escuelas: reducción del tiempo lectivo y, por tanto, pérdida de aprendizajes; desvinculación emocional de algunos alumnos, normalmente de los más vulnerables, con la escuela; e implantación de una enseñanza en un entorno digital, con un aprovechamiento educativo desigual a causa de motivos socioculturales y económicos de las diferentes familias del alumnado. Por otro lado, los efectos derivados de la crisis económica, que genera incertidumbre entre las familias y sus hijos. Y, por último, los efectos derivados del periodo de confinamiento, que pueden acarrear entre los estudiantes problemas de índole psicosocial, como por ejemplo: el estrés, la ansiedad, el aumento de las tasas de maltrato infantil, los problemas de adicciones,... Todos ellos, se concentran en una serie de consecuencias o de 
costos para la Educación y para el aprendizaje de los alumnos más vulnerables a largo plazo (Figura 1). En esa línea, los profesores Valiente-Barroso, Suárez-Riveiro y Martínez-Vicente (2020) han evidenciado a nivel nacional, en un estudio llevado a cabo con 519 alumnos de la Comunidad de Cantabria que cursan Educación Primaria, el impacto de los factores físicos, emocionales y ambientales en el rendimiento académico de los estudiantes durante el confinamiento, mediante el pase de algunos cuestionarios.

FIGURA 1

Impactos que afectan a la Educación

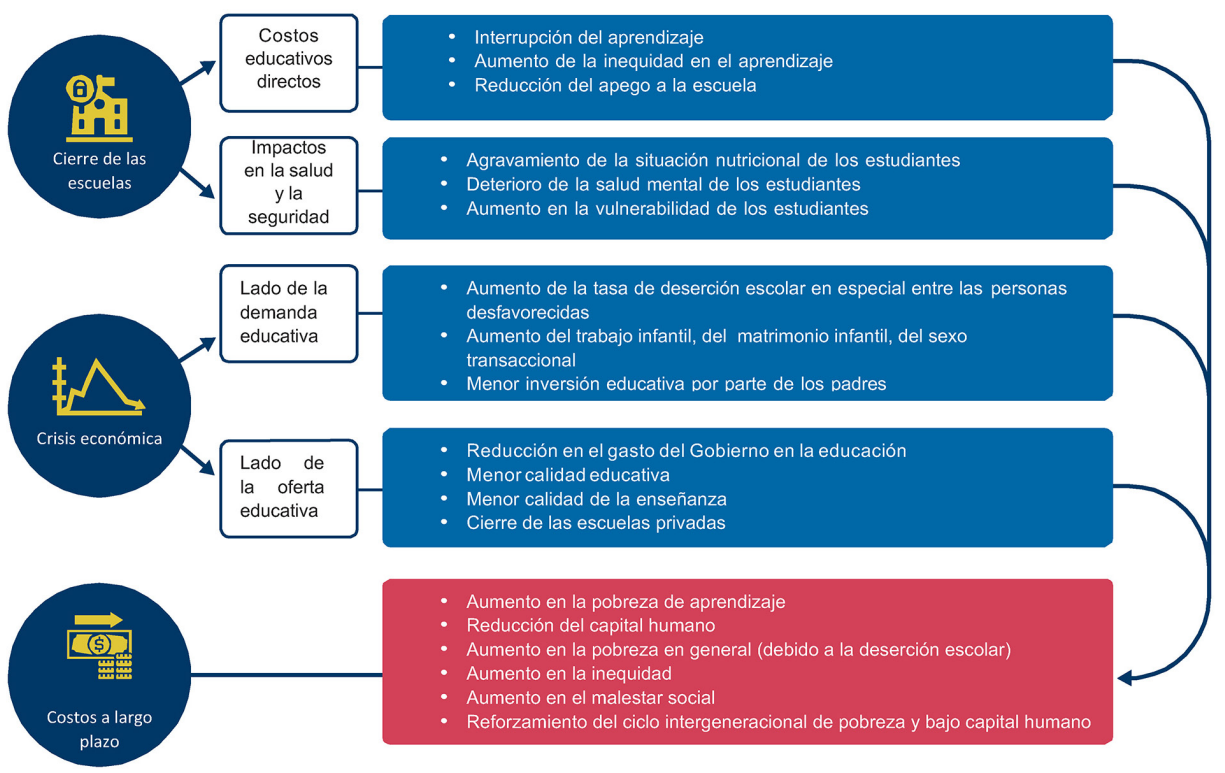

Fuente: Rogers \& Sabarwal (2020, p. 13)

Para intentar dar una respuesta pedagógica a esta situación excepcional, nos hemos planteado en este artículo descifrar y analizar el impacto y la realidad de las tres brechas educativas que se han generado a partir de la COVID-19: la brecha de aprendizaje, la brecha digital y la brecha escolar, tomando como referencia investigaciones tanto nacionales como de organismos internacionales. Posteriormente, se elaboran una serie de propuestas educativas, a nivel nacional, con el fin de reestablecer la equidad en el sistema educativo. La metodología utilizada ha sido la hermenéutica interpretativa, ya que nos permite interpretar y comprender, de manera crítica, la realidad educativa y establecer medidas de intervención adecuadas y adaptadas al contexto educativo. 
ROBERTO SANZ PONCE Y ELENA LÓPEZ LUJÁN

CONSECUENCIAS PEDAGÓGICAS ENTRE EL ALUMNADO DE ENSEÑANZA BÁSICA DERIVADAS DE LA COVID-19. UNA REFLEXIÓN EN TORNO A LOS GRANDES OLVIDADOS DE LA PANDEMIA

\section{LA BRECHA DE APRENDIZAJE: CÓMO LA ESCUELA PUEDE GENERAR DESIGUALDAD DE OPORTUNIDADES}

La realidad demuestra que la brecha de aprendizaje ya existía, a nivel mundial, antes de la pandemia. Según el Informe del Banco Mundial (2020a), en el que se mide el impacto de la COVID-19 en la Educación y se proponen algunas respuestas de política educativa para mitigarlo, ya existían 258 millones de niños y jóvenes excluidos de la escuela previo a la aparición de la COVID-19. También, en muchos países del segundo y del tercer mundo y en algunas zonas del primer mundo se observaba una baja calidad educativa y una preocupante falta de equidad, que afectaba fundamentalmente a los colectivos de alumnos más desfavorecidos, vulnerables, con más carencias y con menos recursos, y que se traducía en menores tasas de aprendizaje, de escolarización y más abandono temprano escolar. La pandemia lo que ha potenciado es su visibilidad, además de generar e incrementar nuevas brechas de aprendizaje en colectivos nuevos y de convertirse en una amenaza real de empeorarlo todo. De esta manera, "la inequidad en el aprendizaje aumentará, dado que solo los estudiantes de familias más acomodadas y educadas tendrán apoyo para seguir aprendiendo en casa” (Banco Mundial, 2020a, p. 5). En el caso de las familias desfavorecidas, «se deja a los niños y las niñas a cargo de su propio aprendizaje o se les asignan otras tareas» (UNESCO, 2020b). Surge así, el concepto de «alumno autosuficiente» (Plá, 2020, p. 34), con todas las consecuencias que esto trae consigo. Por ello, la cuestión cultural de la familia, su nivel de estudios, su implicación en las tareas escolares y en la ayuda y asesoramiento socioemocional de sus hijos,..., se convierten en factores clave de protección ante la situación de confinamiento. La ausencia de todos estos factores se convierte, por tanto, en factor de riesgo que posibilita la aparición de la citada brecha de aprendizaje, «lagunas de aprendizaje» tal y como la denominan Wise, Arden-Hills, Stickney, Butz, Beckleer \& Close (2020), y que normalmente afecta con mayor virulencia a los estudiantes en situación de vulnerabilidad o de riesgo de exclusión social. Véase si no, las diferencias en el aprendizaje en función de los ingresos familiares durante el periodo de confinamiento y la estimación posterior a la apertura de las escuelas.

Del mismo modo, la situación de confinamiento, las condiciones habitacionales de muchos hogares, el acceso a los recursos tecnológicos o las preocupaciones laborales y económicas de muchas familias han podido generar situaciones de estrés y de ansiedad entre los estudiantes. Estas situaciones de estrés y de ansiedad también han tenido consecuencias importantes en el desarrollo educativo, psicológico y emocional de muchos niños y jóvenes (Ballverka et al., 2020; Brooks et al., 2020). Además, pueden acarrear problemas de apego entre los estudiantes y la escuela y generar cierta desvinculación emocional, que aumenta el riesgo de deserción escolar, "pues el apego de los estudiantes vulnerables a la escuela se puede reducir ante la falta de exposición a docentes que los motiven" (Banco Mundial, 2020a, p. 5). Save the Children (2020, p. 4) sitúa al docente como un factor de protección clave frente al abandono escolar del alumnado más vulnerable y afirma que «el 
FiguRA 2

Diferencias en el aprendizaje en función de los ingresos familiares durante el periodo de confinamiento ${ }^{1}$

Un modelo de aprendizaje sobre el confinamiento

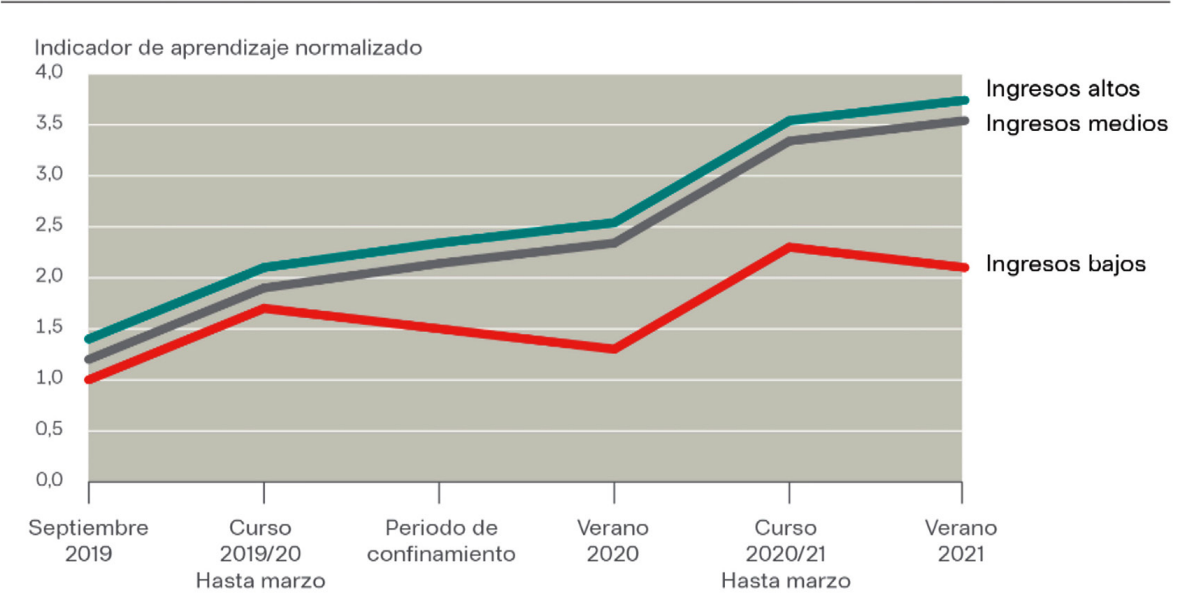

Fuente: Simulación de Cotec a partir de Cooper, Bormain y Fairchild (2010)

Fuente: Zubillaga y Gortazar (2020, p. 8)

trabajo afectivo y emocional, de acompañamiento, escucha y personalización de los procesos educativos que realiza el profesorado [...] queda muy mermado en un momento de especial dificultad para el alumnado más vulnerable». Esta situación también va a afectar a la equidad del sistema educativo.

Aunque, desgraciadamente, tal y como denunciaba Perrenoud (2012, p. 29) esta realidad se viene produciendo ya hace algunos años. La escuela y el sistema educativo en su conjunto tienen una clara razón de ser, que no es otra que la de asegurar la equidad entre sus estudiantes. Fortalecer las capacidades de los más necesitados, dotándoles de mayores herramientas y de mejores recursos para implementar una verdadera igualdad de oportunidades. Así lo expresaba Perrenoud (ídem):

Aquellos a los que la escuela debería preparar mejor para la vida son los que saldrán del sistema educativo sin haber adquirido el nivel de cultura suficiente como para aprender fácilmente en la edad adulta lo que no habrán aprendido en la escuela obligatoria.

1. Esta Figura se elabora por COTEC mediante simulación a partir de los resultados obtenidos por los estudiantes en PISA 2018. Los datos reflejan, por tanto, una previsión de la evolución en el aprendizaje durante un año, tras el confinamiento y cierre de las escuelas sufrido en los meses de marzo-mayo 2020, en España. 
Esta afirmación debería hacernos replantear el sentido, la finalidad y la forma de la educación actual (Sanz y Serrano, 2017).

Además, debemos tomar en consideración lo que los profesores Náñez, Holloway, Jarret \& Kaur (2020) mantienen. Estos docentes, en su última investigación, concluyen que durante el curso todos los alumnos tienen acceso a los recursos necesarios para el aprendizaje, pero que estos recursos se ven radicalmente reducidos durante el confinamiento entre el alumnado más vulnerable, lo que provoca diferencias sustanciales en el aprendizaje. También, el nivel cultural de los padres conlleva que se generen experiencias e interacciones más ricas entre la familia y, por tanto, con respecto a los hijos, lo cual también provoca diferencias en el aprendizaje y en el desarrollo de habilidades y competencias. Por ello, parece lógico pensar, a la luz de los datos (Figura 2), que los niños con más recursos aprenden más allá del aula y de la escuela, realidad que no ocurre con los alumnos con menos recursos y que se ha visto agravado durante los meses de confinamiento (Cáceres-Muñoz et al., 2020).

\section{LA BRECHA DIGITAL: LA EVIDENCIA DEL IMPACTO DE LAS CUESTIONES SOCIOECONÓMICAS EN LA EDUCACIÓN DE NIÑOS Y JÓVENES}

La crisis sanitaria ha puesto de manifiesto el problema de la brecha digital a nivel internacional. Obviamente, no la ha creado, pero ha demostrado que los estudiantes que son hijos de familias con mayores recursos económicos y/o culturales - medido como nivel de estudio de los padres - han tenido muchas más facilidades para poder seguir la educación desde sus hogares (Cabrera, 2020) y, por tanto, mantener un adecuado desarrollo de su aprendizaje durante el periodo de confinamiento. Las diferencias encontradas en función del número de ordenadores en casa en relación con el nivel socioeconómico así lo atestiguan (Figura 3). De esta manera, las familias con más recursos económicos y/o culturales tienen un mejor acceso a internet y una mayor conectividad en sus hogares, así como más conocimientos digitales —alfabetización digital— que las familias más desfavorecidas y/o más vulnerables (Banco Mundial, 2020b). Es evidente que estas diferencias se han visto reflejadas claramente en el desarrollo académico de los estudiantes y en el seguimiento de los estudios durante el periodo de confinamiento, agravando la situación de desigualdad.

El estudio de Gortazar, Moreno y Zubillaga (2020) nos habla de la aparición de 3 brechas de aprendizaje con relación a la brecha digital (García-Fernández, Rivero y Ricis, 2020). Por un lado, encontramos una brecha de acceso de los alumnos a un ordenador, a un espacio concreto y adecuado para el estudio y a una conexión fluida y constante a internet por causas socioeconómicas familiares. La segunda, es una brecha de uso, es decir, de la cantidad de horas destinadas al uso de dichas Tecnologías y a la calidad e idoneidad de las páginas visitadas por los estudiantes, muy en relación con el nivel sociocultural de las familias. Los datos demuestran un mayor uso, en número de horas, aunque de peor calidad, de los alumnos de familias 
FIGURA 3

Número de ordenadores en casa por cuartil socioeconómico ${ }^{2}$

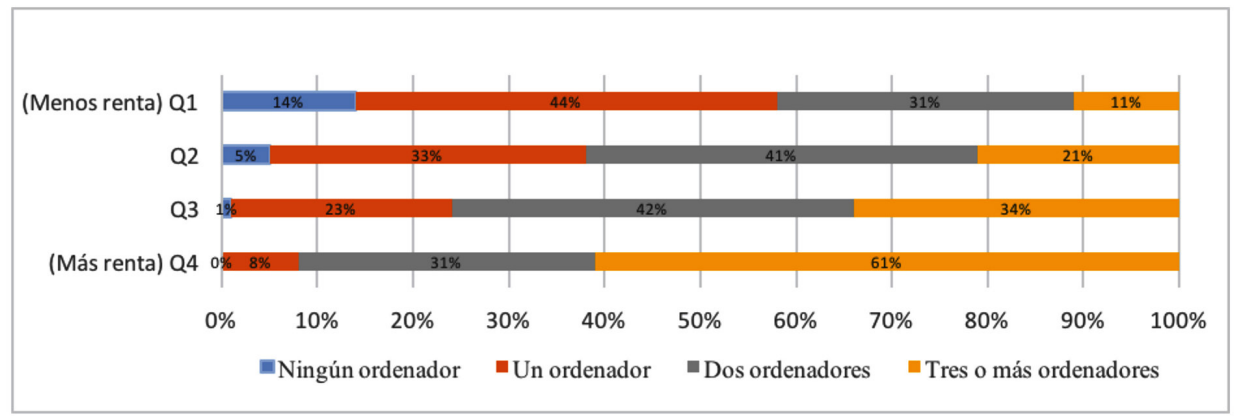

Fuente: Zubillaga y Gortazar (2020, p. 8)

de nivel más bajo en el aspecto socioeconómico. Esta realidad tiene que ver con el nivel de alfabetización digital de las familias, nivel que les permite ayudar a sus hijos, asesorarles y llevar un seguimiento de sus tareas, lo que nuevamente agranda la brecha de aprendizaje entre el alumnado vulnerable. En ese sentido, nuevamente la UNESCO (2020g) mantiene que, con independencia del acceso a la educación a distancia de los alumnos, son varias las condiciones que afectan negativamente a los estudiantes más desfavorecidos, ya que, en muchos casos, dependen del apoyo de unos padres y/o tutores con escasos niveles educativos. Por último, encontramos la brecha escolar o de preparación de las escuelas y de los docentes de la que hablaremos en el siguiente apartado.

Reducir la sensación de distancia y desconexión a través de una enseñanza digital no ha sido conseguido de igual manera en todos los centros escolares, ni con todos los alumnos (Náñez et al., 2020). La falta de preparación técnica y pedagógica de muchos docentes en lo referente a las tecnologías aplicadas a la educación y la falta de medios en muchos centros escolares han imposibilitado hacer realidad este propósito. En España, destaca como el $48 \%$ de los centros escolares no están preparados para llevar a cabo una educación a distancia, frente al 20 \% que hay en Finlandia. Además, el $45 \%$ de los docentes españoles no tienen recursos profesionales eficaces para aprender a utilizar dispositivos digitales y el $47 \%$ no tienen las habilidades técnicas y pedagógicas necesarias para integrar dispositivos digitales en su enseñanza. Si tomamos los datos en función de la titularidad del

2. Esta Figura se elabora por COTEC mediante simulación a partir de los resultados obtenidos por los estudiantes en PISA 2018. Los datos reflejan, por tanto, una previsión de la evolución en el aprendizaje durante un año, tras el confinamiento y cierre de las escuelas sufrido en los meses de marzo-mayo 2020, en España. 
centro, veremos como las diferencias económicas de las escuelas y, por ende, de las familias también influyen en la capacidad de dar una educación de calidad en estas circunstancias. La distribución de los docentes con las habilidades técnicas y pedagógicas necesarias para integrar dispositivos digitales en su enseñanza según el tipo de centro queda así distribuida: centros públicos ( $45 \%$ ), centros concertados (69 \%) y centros privados (76 \%). La distribución de los docentes con los recursos profesionales eficaces para aprender a utilizar dispositivos digitales queda distribuida de la siguiente manera: centros públicos (48\%), centros concertados (70\%) y centros privados (73\%). Por último, la distribución en cuanto a sí los centros disponen de plataformas de teleformación propias, según la titularidad, es: centros públicos (49\%), centros concertados (55\%) y centros privados (71\%) (Gortazar et al., 2020). Estas diferencias también agravan la brecha de aprendizaje en los alumnos con menos recursos.

Para muchos autores (Rodicio, et al., 2020), en España, no se ha adoptado un modelo de educación a distancia, sino que se ha intentado seguir haciendo lo que se hacía presencialmente en el aula utilizando las plataformas digitales. Esto ha conllevado que la brecha digital se convierta en una brecha de aprendizaje. Algunos de los problemas encontrados tienen que ver con la formación del profesorado en TICs y, también, curiosamente, con el nivel de dominio de las tecnologías por parte del alumnado, que aunque haya nacido en la era digital, se ha demostrado que no tenían los conocimientos, destrezas y competencias digitales adecuadas para llevar a cabo una educación a distancia. Por último, el gran problema que se ha detectado durante el periodo de confinamiento y que tiene consecuencias en la actualidad ha sido la atención a la diversidad. La ausencia de recursos y la falta de formación específica de los docentes ha provocado la ausencia de atención a la diversidad. Muchos estudiantes afirman que perder el contacto con el docente y con los compañeros les ha generado dificultades y mantienen que extrañan la pizarra y una atención más personalizada (Babincáková \& Bernard, 2020).

\section{LA BRECHA ESCOLAR: LA CONFIRMACIÓN DE UNA ESCUELA A DIFERENTES VELOCIDADES}

La brecha escolar o de preparación de las escuelas y de los docentes hace referencia a la capacidad de los docentes para activar el proceso de enseñanza-aprendizaje en la «nueva normalidad» y estimular el aprendizaje, recuperar los aprendizajes perdidos y enganchar nuevamente al alumnado. Esta brecha es, en palabras de Fernández-Enguita (2020), la más evitable.

En cuanto a la capacidad de los docentes para hacer frente a la nueva realidad, son muchas las cualidades que debe presentar el profesorado para reenganchar al alumnado hacia el aprendizaje. Tras muchos meses de ausencia de los alumnos en las aulas, tras gran cantidad de situaciones complejas a nivel emocional y relacional, tras la aparición de cierto «desapego» por la escuela, el docente y los contenidos 
académicos, llega el momento de reconstruir y cicatrizar las heridas y volver a la senda del aprendizaje. Más si cabe con aquellos alumnos que han sufrido más las consecuencias del confinamiento y del cierre de las escuelas. Tradicionalmente se habla del "olvido veraniego" para definir la pérdida de conocimientos que sufren los estudiantes tras un periodo, más o menos largo, de ausencia en la escuela. Este «olvido veraniego» tiene un impacto mayor en ciertos colectivos. Por tanto, la reapertura de las escuelas, tras el confinamiento, supone un reiniciar el proceso de enseñanza-aprendizaje, un reenganchar a los alumnos, atendiendo a todas las diferencias que se hayan podido generar, tanto de ritmos como de capacidades, mediante nuevas metodologías. No atender a esta realidad va a favorecer la creación de una escuela a dos velocidades, que atenta contra el principio de equidad. En esa línea, Froment, Bohórquez y García-González (2019) nos hablan de la importancia de la "credibilidad docente» como herramienta para favorecer la comunicación profesor-alumno y generar un mejor entorno de aprendizaje.

Además, en esta «nueva realidad» también es fundamental atender el desarrollo socio-emocional de los estudiantes. Se necesitan docentes emocionalmente inteligentes (Cabello et al., 2010; Barrientos et al., 2020) que sean capaces de interpretar, percibir, evaluar y expresar emociones, que las instauren dentro del proceso de enseñanza-aprendizaje y tengan la capacidad de regular las emociones, tanto positivas como negativas, de su propio alumnado. Además, también se precisan docentes apasionados por su profesión, por la enseñanza y por los alumnos (Jordán y Codana, 2019; Day, 2019). Que escuchen a los estudiantes, se interesen por sus problemas, que estén cerca de ellos, que tengan sentido del humor y que estimulen el placer y el deseo por aprender. Van a ser muchas las consecuencias psicoemocionales que presenten algunos de nuestros alumnos. Consecuencias derivadas del confinamiento y de la propia pandemia, como pueden ser: el estrés, la ansiedad, el miedo, la inseguridad,..., (Cao et al., 2020; Ballverka et al., 2020), y que deben ser atendidas por los docentes y por las escuelas sino queremos, nuevamente, crear una escuela a dos velocidades. Estas dos circunstancias, de aprendizaje y de carácter psicoemocional, suelen afectar con mayor virulencia a los alumnos más vulnerables, de familias con menos recursos y, por tanto, con menos posibilidades de ayudar y compensar dichas desigualdades.

\section{AlgunAS PROPUESTAS EDUCATIVAS URGENTES: LA NECESIDAD DE RECUPERAR LA ESCUELA}

Tras el momento de emergencia educativa que vivimos en el periodo de confinamiento, y que nos llevó a una educación de campaña, de improvisación rápida y poco meditada, para intentar dar una respuesta educativa a la crisis sanitaria, llega el momento de la reconstrucción del sistema educativo. Se trata de pensar en un modelo educativo más inclusivo, sólido, equitativo y resiliente, que preste un apoyo 
especial a los alumnos de familias más desfavorecidas o que han sufrido con mayor virulencia las consecuencias educativas del confinamiento.

Dentro de las respuestas que podemos establecer para paliar estas consecuencias psicopedagógicas, la UNESCO (2020d) anima a estrechar los vínculos y las relaciones entre docentes y familias, y a que ambos participen activamente en las decisiones sobre la idoneidad del curriculum. Mantiene que es momento de repensar el curriculum, de redefinirlo, de "cambiar la rutina normal por soluciones creativas e innovaciones flexibles que apunten a un aprendizaje significativo, relevante, eficaz y de calidad en tiempos de crisis» (UNESCO, 2020d, p. 2). Un curriculum enfocado a la educación en valores, a responder a las necesidades sociales y emocionales de los estudiantes, a mejorar sus habilidades de pensamiento superior, así como desarrollar las habilidades sociales y emocionales de empatía, trabajo en equipo, colaboración, resiliencia, proactividad, iniciativa y conducta responsable entre el alumnado. Algunos países, con alto rendimiento en las evaluaciones internacionales ya habían comenzado políticas de reducción sustancial de contenidos curriculares (Hong Kong y Singapur, son dos ejemplos claros de ello), flexibilizando el currículum e implementando aprendizajes basados en proyectos, problemas o retos, que potencien una enseñanza experiencial frente a otros métodos de corte más memorístico (Banco Mundial, 2018). Hay autores que plantean la necesidad de cambiar el currículum para atender las necesidades de los diferentes, los excluidos, los invisibles y los olvidados, con el objeto de potenciar la igualdad real de oportunidades (De Alba, 2020).

En ese misma línea, con la intención de potenciar la recuperación del aprendizaje, el Banco Mundial (2020a) propone a día de hoy: implementar métodos pedagógicos y planes de estudios focalizados, que desarrollen las competencias blandas: liderazgo, aprender a aprender y a desaprender, innovación, creatividad, resiliencia, empatía, educación emocional y afectiva, trabajo colaborativo,... (López-Martín, 2020); llevar a cabo una enseñanza personalizada, tras una adecuada evaluación de diagnóstico post-confinamiento (UNESCO, 2020e), que nos ayude a situar al alumno y a detectar las posibles carencias y/o retrasos en el aprendizaje que haya podido sufrir durante el tiempo de confinamiento y, así, adaptar la enseñanza a sus distintos ritmos (Rogers \& Sabarwal, 2020); poner al servicio de los alumnos programas de salud mental y emocional, llevados a cabo por especialistas en psicopedagogía, con el objeto de paliar los problemas de estrés, ansiedad, miedo,..., que se hayan podido generar con la pandemia y con el confinamiento; desarrollar programas de formación del profesorado destinados a la preparación metodológica - también en el uso de las tecnologías de la educación-, a la gestión del aula - motivación, resolución de conflictos-, al diagnóstico de necesidades, a la atención a la diversidad, ...; dotar a los sistemas educativos, a las escuelas y al profesorado de los recursos personales y materiales necesarios para poder hacer frente a los desafíos planteados; y llevar a cabo, desde la Inspección Educativa, programas para el diagnóstico rápido de 
los posibles casos de deserción escolar. Todo ello, bajo estrictas medidas sanitarias que aseguren la salud de los estudiantes en los recintos escolares: aulas burbuja, grupos de convivencia, lavado constante de manos, uso de mascarillas, ventilación adecuada,...

Otra de las medidas a llevar a cabo hubiera sido y es la de implementar Programas de Apoyo escolar a las familias en situación de vulnerabilidad (UNICEF, 2020a). Estos Programas deberían haberse puesto en funcionamiento durante el confinamiento, pero también deben desarrollarse post-confinamiento, una vez los alumnos ya han vuelto a las aulas. Las dificultades, los miedos, las situaciones complejas continúan en muchas familias a pesar de la vuelta a la "normalidad educativa» y es la escuela, sus profesionales y sus docentes quiénes deben asesorar y orientar a estas familias. "La crisis de la COVID-19 nos ha recordado la importancia de las escuelas como centros de servicios sociales»(UNESCO, 2020f, p. 15). Además, otra medida a considerar es la planificación sostenible de la enseñanza y de los objetivos a largo plazo de la educación. Para ello, debemos aprender de los aspectos positivos que haya podido traer consigo la educación a distancia e incluirlos y potenciarlos en la nueva educación presencial. No es descartable que se puedan producir nuevos confinamientos en el futuro y debemos prepararnos para dar una respuesta más adecuada, pedagógica, inclusiva, dinámica y justa. Marshall, Shannon \& Love (2020) proponen introducir "días de aprendizaje digital» a lo largo del curso escolar regular, tanto para docentes como para alumnos, con el objetivo de ir acostumbrándolos a esta forma de trabajar ante posibles nuevos confinamientos. Se trata de aprender de los errores para no repetirlos, de revisar el modelo pedagógico imperante y de capacitar al profesorado en las competencias digitales.

A nivel institucional, la escuela post-confinamiento debe convertirse en un centro de resiliencia (Day y Gu, 2015), que prepara y ayuda a los alumnos a superar las dificultades y a empoderarse. Debe ayudar a disminuir los factores de riesgo (estrés, fracaso escolar, ansiedad, problemas en casa,...) y establecer redes de apoyo y factores de protección. Debe surgir una escuela para cada estudiante (Beaudoin, 2013), que atienda sus necesidades personales, pedagógicas y académicas, que personalice la educación como mecanismo fundamental para reenganchar a los estudiantes. Y, por último, se debe implementar, en estos momentos, una escuela a "fuego lento» (Sanz, 2020), que potencie la reflexión pausada, el pensamiento y la discusión frente a la velocidad y fugacidad de un aprendizaje memorístico e irreflexivo. Solo de esta manera, atendiendo a un estilo docente y escolar concreto podremos recuperar a los alumnos más vulnerables, aquellos que han perdido la ilusión y el interés por la escuela y por el aprendizaje.

Todo ello, hace que la tarea docente se complejice, ya que el profesorado debe estar preparado para evaluar los retrasos de aprendizaje de sus alumnos; capacitado para adecuar los contenidos enseñados al nivel y al ritmo de sus estudiantes; tener la habilidad de priorizar en el curriculum todo aquello que es esencial frente 
a lo simplemente importante; tener la visión y la sensibilidad para apoyar a los alumnos en riesgo, vulnerables de ser olvidados o de abandonar el sistema educativo; y, necesariamente, tener una adecuada capacitación digital en el uso de las herramientas pedagógicas online. Es momento de fomentar la "credibilidad docente» para implementar un adecuado estilo comunicativo, basado en la confianza y el respeto mutuo; para gestionar el aula desde un estilo democrático, prosocial y de liderazgo pedagógico; para establecer relaciones cercanas desde el punto de vista psicológico, en un momento, como el actual, donde se han perdido las sonrisas, la proximidad física o el contacto entre personas. Y todo ello, desde la capacidad de inventar nuevas estrategias metodologías, colaborativas y activas desde el respeto a las medidas sanitarias de distanciamiento social. Instaurar el trabajo por Proyectos frente al «abuso» de la lección magistral que se lleva a cabo hoy en día en la escuela. Apostar por un aprendizaje significativo, que prepare para la vida y no solo para superar obstáculos en la escuela. Sustituir una escuela centrada en conocimientos por una escuela competencial, que implemente y desarrolle las competencias básicas y las competencias «blandas» entre el alumnado. Establecer una mayor flexibilidad en el sistema frente a la rigidez actual, que permita a los docentes tomar decisiones — "Capital decisorio» - sobre los contenidos, los agrupamientos, ..., para adecuar la enseñanza a la diversidad de los estudiantes. Dotar de mayores recursos personales en las escuelas para atender al alumnado vulnerable y posibilitar una verdadera igualdad de oportunidades. Y, todo ello, potenciando y estimulando la formación permanente del profesorado para adecuarse a los retos y desafíos planteados en la educación actual.

\section{ConCLUSIONES}

La crisis de la COVID-19, según UNICEF (2020b), es una crisis de los niños. Por ello, debemos poner todos los medios y todos los recursos para evitar una generación pérdida y, en ese sentido, la educación y la escuela son dos factores fundamentales de protección y de compensación de las desigualdades económicas y/o culturales. Pero, debemos repensar el sentido y finalidad de la educación y de la escuela en el contexto actual.

Si no se toman las medidas adecuadas y se mitigan los efectos perversos del cierre de las escuelas y de la pérdida de aprendizaje derivada de dicho cierre, se estima que la "pobreza de aprendizaje» en el escenario post-COVID-19 aumentará en torno a un $10 \%$ en la escuela primaria a nivel mundial (World Bank, 2020). Este concepto de «pobreza de aprendizaje» es definido como la privación de escolaridad — la no asistencia a escuela - y de aprendizaje de calidad, es decir, la no adquisición de las competencias básicas a lo largo de los años de escolarización. Esta realidad agrava la situación de vulnerabilidad de un colectivo concreto: los alumnos con menos recursos. 
Tras la COVID-19 se han roto las certidumbres y el panorama educativo se presenta lleno de inseguridades, de amenazas, de desafíos y de nuevos retos. Por tanto, se debe trabajar en mejorar los entornos educativos, tanto escolares como sociales y familiares, fundamentalmente en el caso de los niños más desfavorecidos. Según Bauman (2017) mucha gente justifica la desigualdad social en base a la idea de que la naturaleza distribuye capacidades diferentes y desiguales entre las personas y, por tanto, que cada uno puede llegar hasta dónde puede llegar. Desgraciadamente, continúa diciendo Bauman, estas diferencias siempre acaban afectando a aquellas personas que tienen menos posibilidades — recursos - de desarrollar plenamente sus capacidades, por eso la escuela debe servir como compensadora social, intelectual y moral. Nadie está destinado al fracaso ni condenado a la exclusión, ya que todos los alumnos tienen la capacidad de aprender y la escuela no puede ni debe anteponer a las élites frente a los alumnos más vulnerables (Meirieu, 2016; 2019). La escuela debe garantizar la igualdad de oportunidades, sabiendo que la igualdad de oportunidades no es dar a todos lo mismo, sino a cada cual aquello que necesita para su correcto desarrollo. Como afirma Hattie (2017, p. 195) no podemos pedir a todos los alumnos que estén igualmente capacitados y motivados para el aprendizaje escolar, ni tampoco podemos pedir a las familias de estos alumnos que los alimenten bien, que les hagan descansar las horas necesarias y que además les ayuden de manera correcta a hacer los deberes. «Esto sería maravilloso, pero el principal papel de la escuela [-no lo olvidemos-] es ayudar a los alumnos a adquirir estos hábitos; [por lo que] no deberíamos discriminar a los alumnos cuyos padres no saben cómo ayudarles a hacerlo.» Esta tarea es una labor propia de las escuelas y de los docentes y, por tanto, es labor nuestra aplicar los mecanismos necesarios para atenuar los efectos derivados de la brecha de aprendizaje, la brecha digital y la brecha escolar.

\section{REFERENCIAS BIBLIOGRÁFICAS}

Babincáková, M. \& Bernard, P. (2020). Online experimentation during COVID-19 Secondary School closures: teaching methods and student perceptions. Journal of Chemical Education, 97, 3295-3300. https://doi.10.1021/acs.jchemed.0c00748

Ballverka, N., Gómez-Benito, J., Hidalgo, M. ${ }^{a}$ D., Gorostiga, A., Espada, J. P., Padilla, J. L., y Santed, M. A. (2020). Las consecuencias psicológicas de la COVID-19 y el confinamiento. Informe de investigación. Universidad País Vasco. https://cendocps.carm.es/documentacion/2020_Consecuencias_psicologicas_COVID19.pdf

Banco Mundial (2018). Educating for the future: the case of East Asia. Education for Global Development. World Bank Blogs. https://blogs.worldbank.org/education/educatingfuture-case-east-asia

Banco Mundial (2020a). COVID-19: Impacto en la Educación y Respuestas de política pública. Banco Mundial. https://www.bancomundial.org/es/topic/education/publication/thecovid19-pandemic-shocks-to-education-and-policy-responses 
Banco Mundial. (2020b). Guidance Note on Education System's Response to COVID-19. Banco Mundial. http://documents1.worldbank.org/curated/en/450881585235950757/ Guidance-Note-on-Education-Systems-Response-to-COVID19.pdf

Banco Mundial. (2020c). La pobreza y la prosperidad compartida 2020: un cambio de suerte. Banco Mundial. https://www.bancomundial.org/es/research/brief/poverty-and-sharedprosperity-2020-reversals-of-fortune-frequently-asked-questions

Barrientos, A., Sánchez, R., y Pericacho, F. J. (2020). Competencias sociales y emocionales del profesorado de Educación Infantil y su relación con la gestión del clima de aula. Estudios sobre Educación, 38, 59-78. https://doi.10.15581/004.38.59-78

Bauman, Z. (2017). ¿La riqueza de unos pocos nos beneficia a todos? Paidós.

Beaudoin, N. (2013). Una escuela para cada estudiante. La relación interpersonal, clave del proceso educativo. Narcea.

Brooks, S. K., Webster, R. K., Smith, L. E., Woodland, L., Wessely, S., Greemberg, N., \& Rubin, G. J. (2020). The psychological impact of quarantine and how to reduce it: rapid review of the evidence. The Lancet, 395(10227), 912-920. https://doi.10.1016/S0140-6736(20)30460-8

Cabello, R., Ruíz-Aranda, D. y Fernández-Berrocal, P. (2010). Docentes emocionalmente inteligentes. Revista Electrónica Interuniversitaria de Formación del Profesorado, 13(1), 41-49.

Cabrera, L. (2020). Efectos del coronavirus en el sistema de enseñanza: aumenta la desigualdad de oportunidades educativas en España. Revista de Sociología de la Educación - RASE, 13(2), 114-139. http://dx.doi.org/10.7203/RASE.13.2.17125

Cáceres-Muñoz, J., Jiménez, A. S., y Martín-Sánchez, M. (2020). Cierre de escuelas y desigualdad socioeducativa en tiempos de COVID-19. Una investigación exploratoria en clave internacional. Revista Internacional de Educación para la Justicia Social, 9(3e), 199-221. https://doi.org/10.15366/riejs2020.9.3.011

Cao, W., Fang, Z., Hou, G., Han, M., Xu, X., Dong, J., \& Zheng, J. (2020). The psychological impact of the COVID-19 epidemic on college students in China. Psychiatry Research, 287, 1-5. https://doi.10.1016/j.psychres.2020.112934

Day, C. (2019). Educadores comprometidos. Qué son, qué hacen, por qué lo bacen y lo que verdaderamente importa. Narcea.

Day, C., y Gu, Q. (2015). Educadores resilientes, escuelas resilientes. Construir y sostener la calidad educativa en tiempos difíciles. Narcea.

De Alba, A. (2020). Currículo y operación pedagógica en tiempos de COVID-19. Futuro incierto. En H. Casanova Cardiel (Coords.), Educación y pandemia. Una visión académica (pp. 289-294). ISSUE. https://issuu.com/educomplexus/docs/educacion_pandemia/97

Fernández Enguita, M. (2020). Una pandemia imprevisible ha traído la brecha previsible. Cuaderno de Campo. https://blog.enguita.info/

FOESSA (2020). Distancia social y Derecho al cuidado. FOESSA. https://www.caritas.es/ producto/distancia-social-y-derecho-al-cuidado/

Froment, F., Bohórquez, M. ${ }^{a}$ R., y García-González, A. J. (2019). Credibilidad docente. Una revisión de la literatura. Teoría de la Educación. Revista Interuniversitaria., 32(1), 23-54. https://doi.10.14201/teri.20313

García-Fernández, N., Rivero, M. ${ }^{a}$ L., y Ricis, J. (2020). Brecha digital en tiempo de COVID-19. Revista Educativa Hekademos, 28, 76-85. https://revistas.uam.es/reice/article/view/10103

Gortazar, L., Moreno, J. M., y Zubillaga, A. (2020). COVID-19 y EDUCACIÓN II: escuela en casa $y$ desigualdad. Un análisis a partir de las respuestas de directores y alumnos recogidos 
en el Informe PISA 2018. COTEC. https://cotec.es/noticia/el-impacto-de-la-escuela-encasa-en-las-brechas/832831a8-38ab-476e-8833-9c2083b89354

Hattie, J. (2017). "Aprendizaje visible» para profesores. Maximizando el impacto en el aprendizaje. Paraninfo.

Jordán, J. A., y Codana, A. (2019). La influencia del profesor apasionado en la mejora académica y el desarrollo personal de sus alumnos. Estudios sobre Educación, 36, 31-51. https://doi.10.15581/004.36

López-Martín, R. (2020). Reflexiones educativas para el post-COVID-19. Recordando el futuro. Revista Internacional de Educación para la Justicia Social, 9(3e), 127-140. https:// doi.10.15366/riejs2020.9.3.007

Marshall, D. T., Shannon, D. M., \& Love, S. M. (2020). How teachers experienced the COVID-19 transition to remote instruction. Kappan, 102(3), 46-50. https:// doi.10.1177/0031721720970702

Meirieu, P. (2016). Recuperar la Pedagogía. De lugares comunes a conceptos claves. Paidós.

Meirieu, P. (2019). Riquezas y límites del enfoque por "competencias» del ejercicio de la profesión docente hoy. Pedagogía y Saberes, 50, 97-108. http://www.scielo.org.co/pdf/ pys/n50/0121-2494-pys-50-97.pdf

Náñez, J. E., Holloway, S. R., Jarret, D. B., \& Kaur, G. (2020). A call to address academic difficulties resulting from the COVID-19. Related change in Education delivery. Word Journal of Education and Humanities, 2(4), 6-10. https://doi.org/10.22158/wjeh.v2n4p6

Perrenoud, P. (2012). Cuando la escuela pretende preparar para la vida. ¿Desarrollar competencias o enseñar otros saberes? Graó.

Plá, S. (2020). La pandemia en la escuela: entre la opresión y la esperanza. En H. Casanova Cardiel (Coords.), Educación y pandemia. Una visión académica (pp. 30-38). ISSUE. https://issuu.com/educomplexus/docs/educacion_pandemia/97

Reimers, F. M., y Schleicher, A. (2020). Orientaciones para guiar la respuesta educativa a la pandemia COVID-19.OECD. https://globaled.gse.harvard.edu/files/geii/files/orientaciones_para_guiar_la_respuesta_educativa_a_la_pandemia_covid-19.pdf

Rodicio, M. ${ }^{a}$ L., Ríos, M. ${ }^{a}$ P., Mosquera, M ${ }^{a}$ J., y Penado, M. (2020). La brecha digital en estudiantes españoles ante la crisis de la COVID-19. Revista Internacional de Educación para la Justicia Social, 9(3e), 103-125. https://doi.10.15366/riejs2020.9.3.006

Rogers, H., y Sabarwal, S. (2020). COVID-19: Impacto en la Educación y respuestas de política pública. Banco Mundial. https://openknowledge.worldbank.org/bitstream/ handle/10986/33696/148198SP.pdf?sequence=6\&isAllowed $=y$

Sanz, R. (2020). Una escuela a fuego lento. En C. Naval, A. Bernal, G. Jover, y J. L. Fuentes (Coords.), Perspectivas actuales de la condición bumana y la acción educativa (pp. 301-313). Dykinson.

Sanz, R., y Serrano, A. (2017). ¿La educación cambia? Repensando el sentido y finalidad de una escuela para todos. Teoría de la Educación. Revista Interuniversitaria, 29(2), 167-184. http://doi.org/10.14201/teoredu292167184

Save the Children (2020). COVID-19: cerrar la brecha. Impacto educativo y propuestas de equidad para la desescalada. Save the Children. https://www.savethechildren.es/actualidad/informe-covid-19-cerrar-la-brecha

UNESCO. (2020a). UNESCO COVID-19 Education Response. Education Sector issue notes. "Supporting teachers and education personnel during times of crisis." UNESCO. https:// 
reliefweb.int/report/world/unesco-covid-19-education-response-education-sector-issuenotes-issue-note-n-81-april

UNESCO. (2020b). Respuesta educativa de la UNESCO a la COVID-19. Notas temáticas del Sector Educación. "Promoción del bienestar socioemocional de los niños y los jóvenes durante la crisis.» UNESCO. https://es.unesco.org/covid19/educationresponse/support

UNESCO. (2020c). Respuesta educativa de la UNESCO a la COVID-19. Notas temáticas del Sector Educación. "Planificación educativa sensible a las crisis.» UNESCO. https://es.unesco. org/covid19/educationresponse/support

UNESCO. (2020d). Respuesta educativa de la UNESCO a la COVID-19. Notas temáticas del Sector Educación. "Mantención de los resultados de calidad en el contexto del aprendizaje remoto.» UNESCO. https://es.unesco.org/covid19/educationresponse/support

UNESCO. (2020e). UNESCO COVID-19 Education Response. Education Sector issue notes. "Distance learning strategies in response to COVID-19 school closures." UNESCO. https:// reliefweb.int/report/world/unesco-covid-19-education-response-education-sector-issuenotes-issue-note-n-81-april

UNESCO. (2020f). La educación en un mundo tras la COVID: nueve ideas para la acción pública. Comisión Internacional sobre los futuros de la Educación. UNESCO. https:// reliefweb.int/report/world/unesco-covid-19-education-response-education-sector-issuenotes-issue-note-n-81-april

UNESCO. (2020g). Inclusión y educación: todos y todas sin excepción. UNESCO. https:// es.unesco.org/gem-report/report/2020/inclusion

UNICEF. (2020a). La Educación frente al COVID-19. Propuestas para impulsar el derecho a la educación durante la emergencia. UNICEF. https://www.unicef.es/educa/biblioteca/ la-educacion-frente-al-covid-19

UNICEF. (2020b). Evitar una generación perdida a causa de la COVID-19. Un plan de seis puntos para responder, recuperarse y reimaginar un mundo para todos los niños después de la pandemia. UNICEF. https://www.unicef.org/es/informes/evitar-una-generacionperdida-a-causa-de-la-covid

Valiente-Barroso, C., Suárez-Riveiro, J. M. y Martínez-Vicente, M. (2020). Rendimiento académico, aprendizaje y estrés en el alumnado de Primaria. Revista Complutense de Educación, 31(3), 365-374. https://doi.10.5209/rced.63480

Zubillaga, A., y Gortazar, L. (2020). COVID-19 y EDUCACIÓN I: problemas, respuestas y escenarios. Documento técnico de análisis de la situación educativa derivada de la emergencia sanitaria. COTEC. https://biblio.ontsi.red.es/cgi-bin/koha/opac-detail. pl?biblionumber $=5948$

Wise, A. E., Arden-Hills, R., Stickney, E. M., Butz, D., Beckleer, A., \& Close, C. N. (2020). The potential impact of COVID-19 on student learning and how schools can respond. Educational Measurement Issues and Practice Fall, 39(3), 60-64. https://doi.org/10.1111/ emip. 12357

World Bank. (2020). Learning poverty in the time of COVID-19: a crisis within a crisis. World Bank. https://openknowledge.worldbank.org/handle/10986/34850 